OPEN ACCESS

Edited by:

Hany M. Ibrahim,

University of Menoufia, Egypt

Reviewed by:

Vikrant Sudan

U.P. Pandit Deen Dayal Upadhyaya Veterinary University, India

Rogério De Jesus,

Federal University of the Recôncavo of Bahia, Brazil

*Correspondence: Xiuhong Zhu Zhuxiuhong001@126.com

Yurong Yang

yangyu7712@sina.com;

yryang@henau.edu.cn

Specialty section: This article was submitted to Parasitology,

a section of the journal

Frontiers in Veterinary Science

Received: 16 August 2021 Accepted: 08 September 2021

Published: 14 October 2021

Citation:

Jiang $N$, Xin S, Zhu N, Yang L, Huang $W, H u$ J, Zhu $X$ and Yang $Y$ (2021) First Report of Sarcocystis Masoni in a Captive Alpaca Nicugna Pacos) From China

Front. Vet. Sci. 8:759252 doi: 10.3389/fvets.2021.759252

\section{First Report of Sarcocystis Masoni in a Captive Alpaca (Vicugna Pacos) From China}

\author{
Nan Jiang ${ }^{1}$, Shilin Xin ${ }^{1}$, Niuping Zhu ${ }^{1}$, Liulu Yang ${ }^{1}$, Wei Huang ${ }^{1}$, Junjie $\mathrm{Hu}^{2}$, Xiuhong Zhu ${ }^{3 \star}$ \\ and Yurong Yang ${ }^{1 *}$
}

${ }^{1}$ College of Veterinary Medicine, Henan Agricultural University, Zhengzhou, China, ${ }^{2}$ School of Biological Sciences, Yunnan University, Kunming, China, ${ }^{3}$ College of Forestry, Henan Agricultural University, Zhengzhou, China

Background: Sarcocystosis is a parasitic disease caused by intracellular protozoan parasite of the genus Sarcocystis. Tissue samples of alpacas $(n=4)$ from Henan province (China) were screened for Sarcocystis spp. infection by histological examination, pepsin digestion, and molecular assays.

Results: Sarcocystis spp. was detected in heart, liver, spleen, lung, and kidney of an alpaca by molecular assays. Many sarcocysts with inflammation responses were observed in this alpaca myocardium, and they showed a high similarity to Sarcocystis masoni by sequence analysis.

Conclusion: This study is the first to demonstrate Sarcocystis spp. infection in alpaca from China. The higher parasite load in the alpaca myocardium indicated that it had contact with an environment contaminated with sporocysts, and that the alpaca was susceptible to Sarcocystis spp.

\section{Keywords: Sarcocystis spp., alpaca, parasite load, food safety, China}

\section{INTRODUCTION}

Sarcocystosis is a parasitic disease caused by intracellular protozoan parasite of the genus Sarcocystis. Its hosts include intermediate and definitive hosts. Intermediate hosts (birds, mammals, reptiles) are infected by ingesting sporozoites shed by a definitive host (typically carnivores) (1). More than 200 recognized Sarcocystis species worldwide can infect domestic and wild animals, causing a significant adverse impact on food health and economic development $(1,2)$.

According to morphological and molecular characteristics, there are two kinds of Sarcocystis spp. in alpaca: macroscopic (the macrocysts of $S$. aucheniae) and microscopic (the microcysts of $S$. masoni) (3-7). There have been many reports about Sarcocystis spp. infection in the alpacas from Africa and South America (1,8-10). However, there is no report of Sarcocystis spp. infection in alpacas from China.

Most animals infected with Sarcocystis spp. have no clinical symptoms, except in the cases of high doses of infection or immunodeficiency of the host, which result in anorexia, fever, anemia, weakness, weight loss, neurological symptoms, diarrhea, and death $(6,7,11)$. Gastroenteritis, nausea, diarrhea, colic, shivering, and breathing problems can occur in humans after eating raw or uncooked meat that contains some species of Sarcocystis, for example S. hommins or S. suihominnis (1). In this article, S. masoni was detected in the myocardium of an alpaca. This is the first report of a Sarcocystis spp. infection in alpaca from China. 


\section{MATERIALS AND METHODS}

\section{Naturally Infected Alpacas and Sampling}

Four dead alpacas (Vicugna pacos) from a zoo were collected in Zhengzhou city, Henan province $\left(33^{\circ} \mathrm{N}, 113.30^{\circ} \mathrm{E}\right)$, China. Both of them were born in this zoo.

Case 1 was a 4 -year-old female, breastfeeding a 3-month-old pup, displaying skin phyma and dyspnea, which suddenly died on June 20, 2020. Case 2 was an adult female with a systemic abscess and a stillborn fetus 1 month before death, which then showed neurological signs and died on August 6, 2020. Case 3 was a 1-year-old male with dyspnea and fever and died on June 4, 2021. Case 4 was an adult male, also displaying dyspnea and died on June 7, 2021. Treatments included azithromycin and multivitamins, which alleviated their clinical symptoms. However, the animals ultimately died. The tissues of case 1 (spleen, liver, lung, heart, kidney, or skin phyma), case 2 (kidney and brain), case 3 (blood, spleen, liver, lung, heart, kidney, and lymphonodus), and case 4 (blood, spleen, liver, lung, heart, kidney, intestine, and lymphonodus) were sent to the pathology laboratory of Henan Agricultural University for pathological diagnosis. These samples were also screened for T. gondii and Sarcocystis spp. infection. However, they all showed negative results for T. gondii by histological examination, pepsin digestion, and molecular assays. By serological assays, only case 3 showed anti- $T$. gondii antibody (modified agglutination test: 1:50 titer).

\section{Light Microscopy Examination}

The squashed myocardium sections of alpacas were directly examined for Sarcocystis spp. If sarcocysts were found, single cyst was separated from the slide. Three tissue pieces from each sample were processed using conventional paraffin histological methods, and $5-\mu \mathrm{m}$-thick sections were prepared and stained with hematoxylin and eosin. The tissue sections were examined for the presence of Sarcocystis spp. under a light microscope (Olympus Bx43, Tokyo, Japan).

\section{Pepsin Digestion Examination}

Myocardium (50 g) samples were homogenized and digested in acidic pepsin individually (12). The digested tissues were examined for Sarcocystis bradyzoites and cysts by light microscopy.

\section{Molecular Analysis of Sarcocystis}

DNA was extracted from the heart, liver, spleen, lung, kidney, brain, lymphonodus, intestine, skin phyma, myocardium digestive juices, and single cyst from the alpacas using a commercial DNA extraction kit (DP304; Tiangen Biotec Company, Beijing, China). PCR amplification of a 900-bp $18 \mathrm{~S}$ rDNA segment was performed using the primer pair SarcoFext (GGTGATTCATAGTAACCGAACG) and SarcoRext (GATTTCTCATAAGGTGCAGGAG) to check the Sarcocystis spp. infection in alpacas (13). In addition, PCR was performed to amplify a segment of the mitochondrial cytochrome $c$ oxidase subunit 1 gene ( $\operatorname{cox} 1$ ) using the specific primer pairs of SF1 (ATGGCGTACAACAATCATAAAGAA) and SR9 (ATATCCATACCRCCATTGCCCAT). The amplified PCR product was approximately 1038 bp $(14,15)$, and both negative and positive controls were included. The PCR products were sent to Shanghai Sangon Company (China) for bi-directional sequencing on an ABI PRISM ${ }^{\mathrm{TM}} 3730$ XL DNA Analyzer using the BigDye Terminator v3.1 Cycle Sequencing Kit (Applied Biosystems, Foster City, CA, USA). The obtained sequences were analyzed by BLAST (GenBank database). The phylogenetic tree of the Sarcocystis spp. was constructed using the neighbor-joining method (Kimura two-parameter model).

\section{RESULTS}

\section{Pathological Findings}

No visible macroscopic sarcocyst was found in the tissues of four alpacas. Acute interstitial pneumonia, septicemia of the spleen, necrosis of hepatocytes, lipofuscin deposition, renal interstitial hemorrhage, acute glomerulonephritis, a large area of congestion in the lungs, and a large number of inflammatory cell infiltration in the myocardium were observed in case 1. Many fusiform or oval sarcocysts were observed in the myocardium and Purkinje fibers. An obvious inflammatory response (lymphocytes, macrophages, and plasma cell infiltration) was observed in the myocardium. Granulation tissue was observed in skin phyma. Pathological results showed that the infection of the Sarcocystis spp. is not the direct cause of the death of this alpaca, but respiratory failure due to acute interstitial pneumonia (Figure 1).

The average sarcocysts load was $79.00 \pm 4.58$ per square centimeter in the myocardium. The cysts size was about 36.48$204.41 \times 31.32-102.01 \mu \mathrm{m}(n=70)$. The cysts can be seen on the smear after digestion by pepsin. The cysts wall was about $2.65 \pm 1.35 \mu \mathrm{m}$ thick $(n=25)$, and short and scattered villar protrusions were visible. The size of the bradyzoites was about 14-20 $\times 4-5 \mu \mathrm{m}(n=10)$ (Figure 1).

For case 2, tissues (kidney and brain) were stored at $-20^{\circ} \mathrm{C}$ for 2 weeks before being sent to the pathology laboratory of Henan Agricultural University. Thus, detailed pathological information could not be obtained. Both case 3 and case 4 showed acute interstitial pneumonia.

\section{PCR Identification of Sarcocystis spp. Infection}

DNA from the samples of heart, liver, spleen, lung, kidney, single cysts, and pepsin-digested myocardium of case 1 had amplified fragments of approximately 900 bp by primers of 18S rRNA (Supplementary Figure). By primers of cox1, except kidney, fragments of 1,038 bp was also amplified from these samples (Supplementary Figure), indicating the presence of Sarcocystis spp. The DNA from the samples of case 2, case 3, and case 4 was negative for Sarcocystis spp. Sequence analysis was performed on the PCR products from heart, liver, spleen, single cyst, and digestive fluid of case 1 . The nucleotide sequences from the sarcocysts of the alpaca were submitted to GenBank (for 18S rRNA: accession number MW481703, MW481704, for cox 1: accession number PRJNA759055). BLAST results showed that the most similar sequences for MW481703, MW481704 in GenBank were S. masoni KU527113.1, KU527112.1, KU527111.1, KU527110.1, KU527109.1, and KU527108.1 (100\% identity) (Figure 2). 


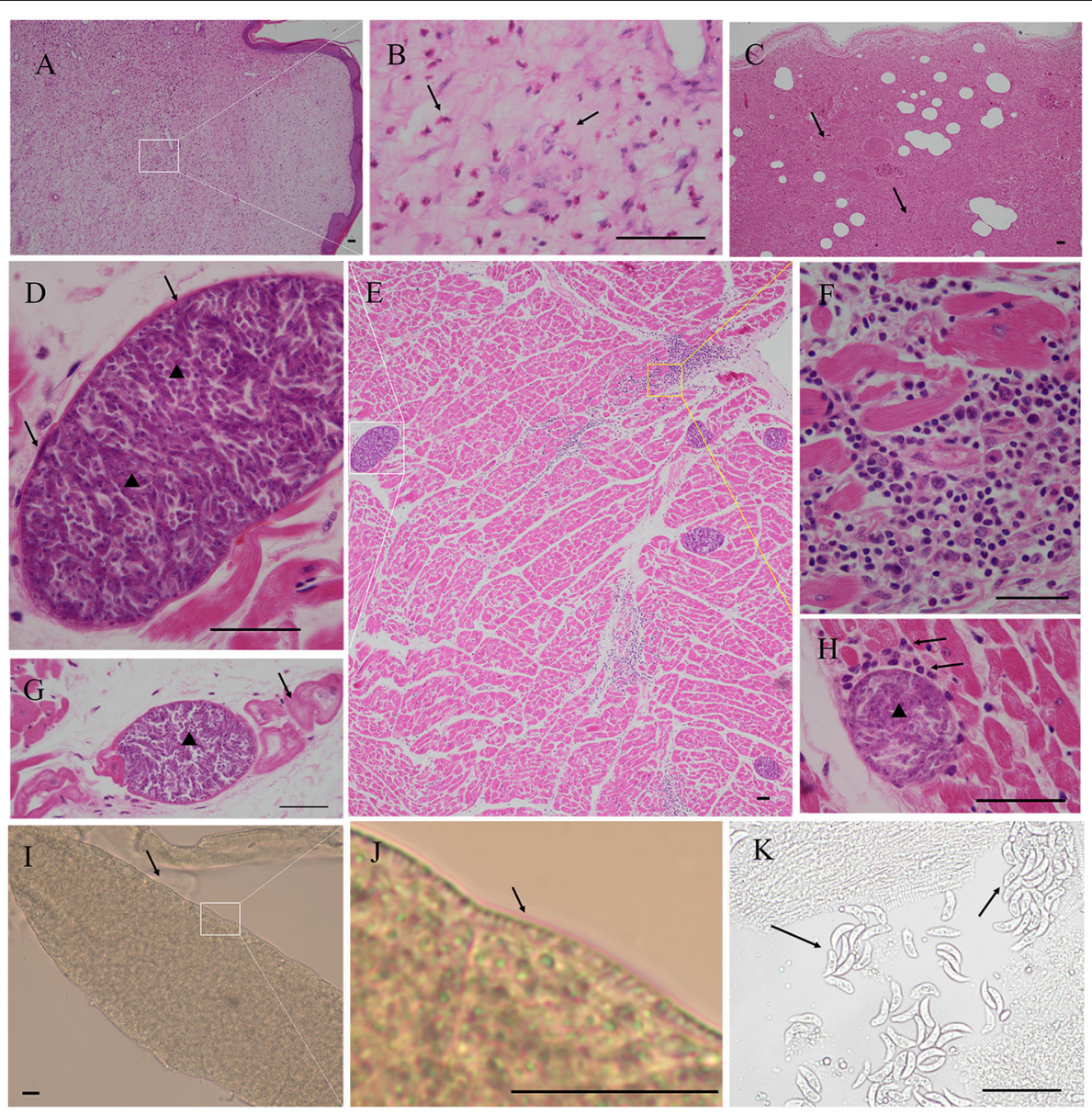

FIGURE 1 | Pathological findings in the myocardium of case 1 alpaca (Vicugna pacos). (A) Granulation tissue was observed in skin phyma. (B) Magnification of picture A; note the presence of numerous eosinophils (arrow), skin phyma. (C) Severe interstitial pneumonia, necrosis, and exudation were observed in the lung (arrow). (D) Magnification of picture E; Sarcocystis masoni cysts were observed in the myocardium, the sarcocyst was separated by septa and formed several rooms, and the bradyzoites were arranged in packets (arrowhead), thin wall (arrow). (E) Presence of numerous S. masoni cysts in the myocardium. (F) Magnification of picture E, myocardial necrosis, and many lymphocytes, macrophages, and plasma cell infiltration. (G) S. masoni (arrowhead) in Purkinje fibers (arrow). (H) Inflammatory responses (arrow) around the degenerated S. masoni sarcocyst (arrowhead) in the myocardium and mononuclear cell infiltration (arrow). (I) S. masoni sarcocyst was found in the pepsin digestion liquid. Thin wall, unstained. (J) Magnification of picture I; short and scattered villar protrusions were visible. (K) S. masoni bradyzoites in the pepsin digestion liquid, unstained. Hematoxylin-eosin (H, E) staining; bar $=50 \mu \mathrm{m}$.

PRJNA759055 sequence had similarity to S. rommeli hosted by cattle, accession numbers KY120286.1, with only $87 \%$ pairwise identity.

\section{DISCUSSION}

Sarcocystis infection is common in many species of animals worldwide, including China. Sarcocysts could be found in skeletal muscles and myocardium $(1,16)$, smooth muscles, and the central nervous system $(1,17)$. They have an adverse effect on the livestock industry, food health, and animal reproduction. However, there is no vaccine to protect animals against clinical sarcocystosis.

Studies have reported antibodies against T. gondii in 30\% and $44 \%$ of llamas from Peru and the USA (18-20) and in 9\% of alpacas from China (21). In this study, antibodies to $T$. gondii (modified agglutination test: 1:50 titer) were found in case 3 alpaca.

Alpacas are raised on a large scale in South America, Africa, Australia, Europe and the United States. Their meat and wool have economic value $(10,22,23)$. Macroscopic (S. aucheniae) and microscopic (S. masoni) sarcocysts were identified in alpacas. Canids are potential definitive hosts $(1,5)$. S. aucheniae appeared in skeletal muscles, and S. masoni were commonly found in the cardiac muscle of alpaca. However, little is known of clinical sarcocystosis in alpaca. Severe myositis was reported in an alpaca (6). Human consumption of Sarcocystis-infected meat (raw or undercooked beef or pork) may lead to gastroenteritis and diarrhea $(1,22)$.

Compared to $18 \mathrm{~S}$ rRNA, cox 1 was found better in differentiation of closely related but morphologically indistinguishable Sarcocystis spp. from cattle $(24,25)$. In this study, BLAST results showed that the most similar sequences 


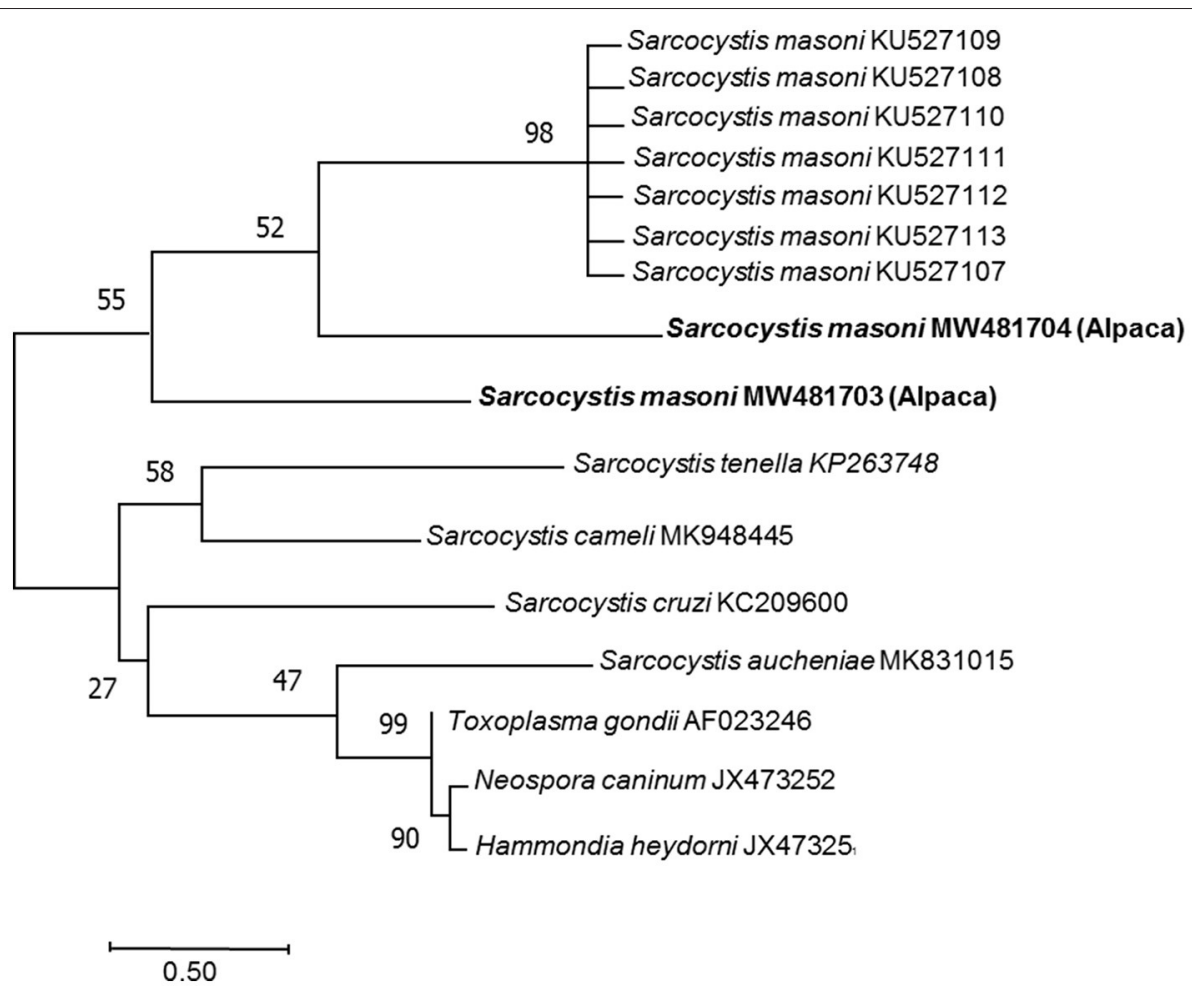

FIGURE 2 | Phylogenetic tree among the Sarcocystis masoni from the alpaca identified in this study and other Sarcocystis spp. The phylogeny was inferred from the neighbor-joining analysis of the $18 \mathrm{~S}$ rRNA sequences based on the distances calculated using the Kimura two-parameter model. Bootstrap values $\mathrm{N}>50 \%$ from 1,000 replicates are shown at the nodes. Sarcocystis spp. (MW481703, MW481704) close relationship to Sarcocystis masoni (KU527107, KU527108, KU527109, KU527110, KU527111, KU527112, and KU527113).

for Sarcocystis spp. from alpaca were S. masoni (100\% identity) by $18 \mathrm{~S}$ rRNA and $S$. rommeli ( $87 \%$ identity) by cox 1 gene. Further, recent research found that cox 1 gene from camels in Saudi Arabia showed low similarity to two Sarcocystis spp. (S. aucheniae or S. masoni) from camels and suggests identification of Sarcocystis spp. with more taxa and different molecular markers, including 18S rRNA and cox 1 (15). Mohamed et al. (26) also suggested using the hybrid approach to characterize the microbe, including Next-Generation Sequencing and conventional PCR combined with Sanger sequencing, and also using RFLP-PCR for characterization of Sarcocystis isolated from camel (27).

Alpacas in China are mainly imported from Australia. Then, most of them are bred in zoos, while some are kept at home as pets. In this study, all the four cases of alpacas were bred in zoo. According to the sarcocyst's morphology, location, host, and molecular characteristics, the sarcocysts in cardiac muscle of alpaca maybe S. masoni. S. masoni nucleotides (18S rRNA) were amplified in the heart, liver, spleen, lung, and kidney of the case 1 alpaca (Supplementary Figure). This indicated that except for the striated muscles, $S$. masoni could grow or exist in other tissues, providing molecular evidence of the S. masoni infection in other tissues. However, the life cycle of $S$. masoni in the intermediate host and definitive host is unknown.

Both case 1 and case 2 alpacas showed skin granulation tissue or systemic abscess. These findings agree with a previous report of an alpaca with a case history of multiple subcutaneous abscesses (6). More investigation is needed to confirm whether sarcocystosis is associated with multiple abscesses in alpacas.

Although parasite loads from different hosts are not comparable. The average myocardial sarcocyst load in alpaca was 79.00 per square centimeter, which is much higher than in beef $\left(1.40\right.$ cysts $\left./ \mathrm{cm}^{2}\right)$ and feral pigs $\left(3.03 \mathrm{cysts} / \mathrm{cm}^{2}\right)$ from the United States and mutton $\left(7.84 \mathrm{cysts} / \mathrm{cm}^{2}\right)$, sheep $(18.07$ cysts $\left./ \mathrm{cm}^{2}\right)$, and cattle $\left(7.1 \mathrm{cysts} / \mathrm{cm}^{2}\right)$ from China (28-32). In this study, the higher parasite load in the alpaca indicated that it had contact with an environment contaminated with sporocysts, and that the alpaca was susceptible to $S$. masoni. Poor sanitation and contact with dogs are considered risk factors for Sarcocystis spp. infection in alpacas (10). For alpaca meat, boiling $\left(100^{\circ} \mathrm{C}\right.$ for $10 \mathrm{~min})$, baking $\left(105^{\circ} \mathrm{C}\right.$ for $\left.65 \mathrm{~min}\right)$, and freezing $\left(-20^{\circ} \mathrm{C}\right.$ for 10 days) could inactivate the Sarcocystis spp. and reduce its risk for other animals (33). This work is the first report of Sarcocystis spp. infection in alpaca from China. It is necessary to further explore the natural clinical cases of sarcocystosis to determine the infection source and the definitive host.

\section{CONCLUSION}

This study is the first to demonstrate Sarcocystis spp. infection in alpaca from China. The higher parasite load in the alpaca myocardium indicated that it had contact with an 
environment contaminated with sporocysts, and that the alpaca was susceptible to Sarcocystis spp. Further study will focus the possible route of infection of these animals, ultrastructure of the cysts (walls and protrusions), and molecular characteristics with more markers of these parasites.

\section{DATA AVAILABILITY STATEMENT}

The datasets presented in this study can be found in online repositories. The names of the repository/repositories and accession number(s) can be found below: https://www.ncbi.nlm. nih.gov/genbank/, MW481703, MW481704.

\section{ETHICS STATEMENT}

The animal study was reviewed and approved by the Institutional Animal Use Protocol Committee of the Henan Agricultural University, China.

\section{REFERENCES}

1. Dubey JP, Calero-Bernal R, Rosenthal BM, Speer CA, Fayer R. Sarcocystosis of Animals and Humans. Boca Raton, FL: CRC Press, Taylor \& Feancis Group (2016). p. 1-481. doi: 10.1201/b19184

2. Tenter AM. Current research on Sarcocystis species of domestic animals. Intl J Parasitol. (1995) 25:1311-30. doi: 10.1016/0020-7519(95)00068-D

3. Saeed MA, Rashid MH, Vaughan J, Jabbar A. Sarcocystis in South American camelids: the state of play revisited. Parasit Vectors. (2018) 11:146. doi: 10.1186/s13071-018-2748-1

4. Regensburger C, Gos ML, Ctibor J, More G. Morphological and molecular characteristics of Sarcocystis aucheniae isolated from meat of Guanaco (Lama guanicoe). J Food Qual Hazards Control. (2015) 2:118-21.

5. More G, Regensburger C, Gos ML, Pardini L, Verma SK, Ctibor J, et al. Sarcocystis masoni, n. sp (Apicomplexa: Sarcocystidae), and redescription of Sarcocystis aucheniae from llama (Lama glama), guanaco (Lama guanicoe) and alpaca (Vicugna pacos). Parasitology. (2016) 143:61726. doi: 10.1017/S003118201600007X

6. Gabor M, Gabor LJ, Srivastava M, Booth M, Reece R. Chronic myositis in an Australian alpaca (Llama pacos) associated with Sarcocystis spp. J Vet Diagn Invest. (2010) 22:966-9. doi: 10.1177/1040638710022 00620

7. La Perle KM, Silveria F, Anderson DE, Blomme EA. Dalmeny disease in an alpaca (Lama pacos): sarcocystosis, eosinophilic myositis and abortion. J Comp Pathol. (1999) 121:287-93. doi: 10.1053/jcpa.1999.0321

8. Gorman TR, Alcaino HA, Munoz H, Cunazza C. Sarcocystis sp. in Guanaco (Lama guanicoe) and effect of temperature on its viability. Vet Parasitol. (1984) 15:95-101. doi: 10.1016/0304-4017(84)90025-6

9. Rooney A, Limon G, Vides H, Cortez A, Guitian J. Sarcocystis spp. in llamas (Lama glama) in Southern Bolivia: a cross-sectional study of the prevalence, risk factors and loss in income caused by carcass downgrades. Prev Vet Med. (2014) 116:296-304. doi: 10.1016/j.prevetmed.2013. 11.014

10. Romero S, Carletti T, Franco CD, More G, Schnittger L, FlorinChristensen M. Seropositivity to Sarcocystis infection of llamas correlates with breeding practices. Vet Parasitol Reg Stud Reports. (2017) 10:6570. doi: 10.1016/j.vprsr.2017.08.006

11. Cornejo BR, Chávez VA, Leyva VV, Falcón PN, Panez LS, Ticona SD. Relationship between the size of macrocysts of Sarcocysistis aucheniae and its viability in Canis familiaris. Rev Inv Vet Perú. (2007) 18:76-83.

12. Dubey JP. Toxoplasmosis of Animals and Humans. 2nd ed. Boca Raton, FL: CRC Press (2010). p. 1-313.

\section{AUTHOR CONTRIBUTIONS}

NJ performed the data analysis and wrote the manuscript. SX, $\mathrm{NZ}$, LY, and WH helped in collecting samples. JH and XZ helped in the revision of the manuscript. YY designed the experiment and wrote the manuscript. All authors have read and approved the final version of the manuscript.

\section{FUNDING}

This study was financed by the Natural Science Foundation of Henan Province, China (202300410214) and the Key research projects of Henan higher education institutions (21A230009).

\section{SUPPLEMENTARY MATERIAL}

The Supplementary Material for this article can be found online at: https://www.frontiersin.org/articles/10.3389/fvets. 2021.759252/full\#supplementary-material

13. More G, Schares S, Maksimov A, Conraths FJ, Venturini MC, Schares G. Development of a multiplex real time PCR to differentiate Sarcocystis spp. affecting cattle. Vet Parasitol. (2013) 197:85-94. doi: 10.1016/j.vetpar.2013.04.024

14. Gjerde B. Phylogenetic relationships among Sarcocystis species in cervids, cattle and sheep inferred from the mitochondrial cytochrome c oxidase subunit I gene. Int J Parasitol. (2013) 43:579-91. doi: 10.1016/j.ijpara.2013.02.004

15. Metwally DM, Al-Otaibi TT, Al-Turaiki IM, El-Khadragy MF, Alajmi RA. Identification of Sarcocystis spp in one-humped camels (camelus dromedarius) from riyadh and dammam, Saudi Arabia, via histological and phylogenetic approaches. Animals. (2020) 10:1108. doi: 10.3390/ani10071108

16. Sazmand A, Joachim A. Parasitic diseases of camels in Iran (1931-2017) - a literature review. Parasite. (2017) 24:21. doi: 10.1051/parasite/2017024

17. Fitzgerald SD, Janovitz EB, Kazacos KR, Dubey JP, Murphy DA. Sarcocystosis with involvement of the central nervous system in lambs. J Vet Diagn Invest. (1993) 5:291-6. doi: 10.1177/104063879300500230

18. Chavez-Velasquez A, Alvarez-Garcia G, Gomez-Bautista M, Casas-Astos E, Serrano-Martinez E, Ortega-Mora LM. Toxoplasma gondii infection in adult llamas (Lama glama) and vicunas (Vicugna vicugna) in the Peruvian Andean region. Vet Parasitol. (2005) 130:93-7. doi: 10.1016/j.vetpar.2005.03.023

19. More G, Pardini L, Basso W, Marín R, Bacigalupe D, Auad G, et al. Seroprevalence of Neospora caninum, Toxoplasma gondii and Sarcocystis sp. in llamas (Lama glama) from Jujuy, Argentina. Vet Parasitol. (2008) 155:158-60. doi: 10.1016/j.vetpar.2008.04.003

20. Dubey JP, Rickard LG, Zimmerman GL, Mulrooney DM. Seroprevalence of Toxoplasma gondii in llamas (Lama glama) in the northwest USA. Vet Parasitol. (1992) 44:295-8. doi: 10.1016/0304-4017(92)90125-S

21. Li J, Ma YT, Liang QL, Li RL, Zheng FG, Liu Q, et al. Serological evidence of toxoplasma gondii and Chlamydia infection in alpacas (Vicugna pacos) in Shanxi Province, northern China. Microb Pathog. (2020) 149:104399. doi: 10.1016/j.micpath.2020.104399

22. Leguía G. The epidemiology and economic impact of llama parasites. Parasitology Today. (1991) 7:54-6. doi: 10.1016/0169-4758(91)90190-Y

23. McGregor B. Production, attributes and relative value of alpaca fleeces in southern Australia and implications for industry development. Small Rumin Res. (2006) 61:93-111. doi: 10.1016/j.smallrumres.2005.07.001

24. Sudan V, Shanker D, Paliwal S, Kumar R, Singh A. Phylogenetics of Sarcocystis fusiformis isolates based on 18S rRNA and cox 1 genes. Microb Pathog. (2021) 159:105144. doi: 10.1016/j.micpath.2021.105144

25. Gjerde B, Hilali M, Mawgood SA. Molecular characterisation of three regions of the nuclear ribosomal DNA unit and the mitochondrial coxl gene 
of Sarcocystis fusiformis from water buffaloes (Bubalus bubalis) in Egypt. Parasitol Res. (2015) 114:3401-13. doi: 10.1007/s00436-015-4566-0

26. Mohamed WMA, Ali AO, Mahmoud HYAH, Omar MA, Chatanga E, Salim B, et al. Exploring prokaryotic and eukaryotic microbiomes helps in detecting tick-borne infectious agents in the blood of camels. Pathogens. (2021) 10:351. doi: 10.3390/pathogens10030351

27. Motamedi GR, Dalimi A, Nouri A, Aghaeipour K. Ultrastructural and molecular characterization of Sarcocystis isolated from camel (Camelus dromedarius) in Iran. Parasitol Res. (2011) 108:949-54. doi: 10.1007/s00436-010-2137-y

28. Calero-Bernal R, Verma SK, Oliveira S, Yang Y, Rosenthal BM, Dubey JP. In the United States, negligible rates of zoonotic sarcocystosis occur in feral swine that, by contrast, frequently harbour infections with Sarcocystis miescheriana, a related parasite contracted from canids. Parasitology. (2015) 142:549-56. doi: 10.1017/S0031182014001553

29. Pritt B, Trainer T, Simmons-Arnold L, Evans M, Dunams D, Rosenthal BM. Detection of Sarcocystis parasites in retail beef: a regional survey combining histological and genetic detection methods. J Food Prot. (2008) 71:21447. doi: $10.4315 / 0362-028 X-71.10 .2144$

30. Dong H, Lu YY, Wang YH, Feng YJ, Dang SY, Liang HD, et al. Histopathology and typing of Sarcocystis in myocardium of cattle and sheep. Chin J Vet Sci. (2018) 38:160-4. doi: 10.16303/j.cnki.1005-4545.2018.01.25

31. Dong H, Su RJ, Wang YH, Tong ZX, Zhang LX, Yang YR, et al. Sarcocystis species in wild and domestic sheep (Ovis ammon and Ovis aries) from China. BMC Vet Res. (2018) 14:377. doi: 10.1186/s12917-018-1712-9
32. Yang YR, Dong H, Su RJ, Wang YH, Wang RH, Jiang YB, et al. High prevalence of Sarcocystis spp. infections in cattle (Bos taurus) from central China. Parasitol Int. (2018) 67:800-4. doi: 10.1016/j.parint.2018.08.006

33. Vilca LM, Durán OJ, Ramos DD, Lucas LJ. Sanitation and detoxification of alpaca (Vicugna pacos) meat with sarcocystiosis though smoked and cured. Revista investigation Veterinary Peru. (2013) 24:537-43.

Conflict of Interest: The authors declare that the research was conducted in the absence of any commercial or financial relationships that could be construed as a potential conflict of interest.

Publisher's Note: All claims expressed in this article are solely those of the authors and do not necessarily represent those of their affiliated organizations, or those of the publisher, the editors and the reviewers. Any product that may be evaluated in this article, or claim that may be made by its manufacturer, is not guaranteed or endorsed by the publisher.

Copyright ( 2021 Jiang, Xin, Zhu, Yang, Huang, Hu, Zhu and Yang. This is an open-access article distributed under the terms of the Creative Commons Attribution License (CC BY). The use, distribution or reproduction in other forums is permitted, provided the original author(s) and the copyright owner(s) are credited and that the original publication in this journal is cited, in accordance with accepted academic practice. No use, distribution or reproduction is permitted which does not comply with these terms. 